\title{
Identification of candidate drugs for the treatment of metastatic osteosarcoma through a subpathway analysis method
}

\author{
XIN LI, MING-LAN YAN and QIAN YU \\ Department of Pharmacy, China-Japan Union Hospital of Jilin University, Changchun, Jilin 130033, P.R. China
}

Received October 8, 2015; Accepted December 6, 2016

DOI: $10.3892 / 01.2017 .5953$

\begin{abstract}
Osteosarcoma (OS) is the third most frequent type of cancer in adolescents and represents $>56 \%$ of all bone tumors. In addition, metastatic OS frequently demonstrates resistance to conventional chemotherapy; thus, the development of novel therapeutic agents for the treatment of patients with metastatic OS is warranted. In the present study, the metabolic mechanisms underlying OS metastasis were investigated using a subpathway analysis method and lead to the identification of candidate drugs for the treatment of metastatic OS. Using the GSE14827 microarray dataset from the Gene Expression Omnibus database, 546 differentially expressed genes were identified between samples from patients with OS who did or did not develop metastatic OS. Furthermore, nine significantly enriched metabolic subpathways were identified, which may be involved in OS metastasis. Finally, using an integrated analysis of metastatic OS-associated subpathways and drug-affected subpathways, 98 small molecule drug candidates capable of targeting the metastatic OS-associated subpathways were identified. This method identified existing anti-cancer drugs, including semustine, in addition to predicting potential drugs, such as lansoprazole, for the treatment of metastatic OS. Transwell and wound healing assays demonstrated that lansoprazole reduced the invasiveness and migration of U2OS cells. These small molecule drug candidates identified through a bioinformatics approach may provide insights into novel therapy options for the treatment of patients with metastatic OS.
\end{abstract}

\section{Introduction}

Osteosarcoma (OS) is the third most frequent type of cancer in adolescents and represents $>56 \%$ of all bone tumors (1). The median age of patients with OS is 16 years old, with a male predominance $(2,3)$. The high incidence of OS during the

Correspondence to: Dr Qian Yu, Department of Pharmacy, China-Japan Union Hospital of Jilin University, 126 Xiantai Street, Changchun, Jilin 130033, P.R. China

E-mail: zrlyyy_yq@163.com

Key words: metabolic subpathways, network, drugs, metastatic osteosarcoma adolescent growth spurt indicates there is an association between this disease and bone development $(4,5)$. The introduction of preoperative high-dose combined chemotherapy in the last three decades has significantly improved the disease-free 5-year survival rate of young patients ( $<40$ years old) to $\sim 50 \%$ (6). However, OS is highly aggressive and numerous patients with OS develop metastases, primarily in the lung, even following resection of the primary tumor $(2,7)$. Furthermore, metastatic OS frequently exhibits resistance to conventional chemotherapies that were effective for treatment of the primary tumor and $>30 \%$ of metastatic OS cases do not respond to chemotherapy $(4,8,9)$. The chemoresistance of malignant OS limits the effectiveness of current cytotoxic drugs (10). Therefore, elucidation of the mechanisms underlying the metastasis of OS and the development of novel drugs to overcome chemoresistance in this disease are warranted to improve the survival rate of patients with OS (1).

The development of novel drugs is a time-consuming and labor-intensive process. Drug repositioning, which explores potential novel uses for known drugs, has become an effective and innovative approach to the drug development process, particularly with the development of system biology and availability of biochemical information in public databases (10). For example, the Gene Expression Omnibus (GEO) database and the Connectivity Map (CMap) database have provided numerous microarray datasets under disease or drug-induced conditions (11).

In the present study, a bioinformatics method based on metabolic subpathway analysis was used to identify potential drugs for the treatment of metastatic OS. Differentially expressed genes (DEGs) between patients with OS who relapsed and those who did not were identified. In addition, existing small molecule drugs capable of targeting metabolic subpathways associated with OS metastasis were considered as potential novel agents for the treatment of metastatic OS. Furthermore, it was experimentally verified that lansoprazole could inhibit the invasion of U2OS cells. The candidate drugs identified by the approach used in the current study may improve the survival of patients with metastatic OS in the future.

\section{Materials and methods}

Microarray data and DEG analysis. The microarray dataset GSE14827 was downloaded from the GEO database (National Center of Biotechnology Information, Bethesda, MD, USA; 
www.ncbi.nlm.nih.gov/geo/query/acc.cgi?acc=GSE14827). The dataset included biopsy samples from 9 patients with OS who developed pulmonary metastases $\leq 4$ years following neoadjuvant chemotherapy and curative resection, and 18 patients who did not relapse in this time frame (7). All tumor samples in the dataset were obtained through diagnostic incisional biopsies from primary sites of OS prior to neoadjuvant chemotherapy at the National Cancer Center Hospital (Tokyo, Japan) between March 1996 and September 2007 (7).

Raw microarray data and probe annotation files from the dataset were downloaded for analysis. The data downloaded included the expression levels of 54,613 probe sets across 27 samples from patients with OS (age, 8-38 years; 13 males and 14 females) that were analyzed using the GeneChip ${ }^{\circledR}$ Human Genome U133 Plus 2.0 array (Affymetrix, Inc., Santa Clara, CA, USA). In the present study, the fold change values for each probe were determined. The fold change value for each probe is the average expression value of OS samples divided by that of normal samples. Subsequently, each probe was converted into an Entrez Gene ID (www.ncbi.nlm.nih.gov/ gene). If a gene mapped onto $>1$ probe, the mean expression value of all corresponding probes was considered the expression value of the gene. Genes with a fold change value of $>1.5$ or $<0.667$ were identified as DEGs.

Identification of subpathways associated with OS metastasis. The Subpathway Miner R package (version 1.0; cran.r-project. org/src/contrib/Archive/SubpathwayMiner), which is a flexible subpathway identification software, was used to obtain subpathways associated with OS metastasis (12). DEGs between patients with OS who did or did not develop metastases were imported into Subpathway Miner, which identified significantly enriched subpathways using hypergeometric tests (12). This software converts pathway structure data from the Kyoto Encyclopedia of Genes and Genomes (KEGG) database (www.genome.jp/ kegg) into undirected R graph objects. In the pathways, proteins are considered nodes and any two nodes belonging to the same reaction are connected by an edge. Finally, Subpathway Miner divides the entire pathway into subpathways using the 'k-clique' method, which is defined as a sub-graph in which the distance between any two nodes is $<\mathrm{k}(12)$. In the present study, $\mathrm{k}$ was set at 3 , thus the distance between proteins in a single subpathway was $<3$. $\mathrm{P}<0.05$ was considered to indicate a statistically significant subpathway.

Analysis of the association between the drugs and metabolic subpathways identified. The global associations between drugs and metabolic subpathways were obtained from a study performed by Li et al (13), in order to identify drugs that serve a role in OS metastasis-associated subpathways. In the above study (12), microarray data from cancer cells treated with or without small molecule drugs were obtained from the CMap database (Broad Institute, Cambridge, MA, USA) (10) and DEGs were identified using a fold change threshold of $>2$ or $<0.05$. Subsequently, drug-affected subpathways were identified for each drug if the corresponding drug-affected genes could be significantly $(\mathrm{P}<0.01)$ enriched in the subpathways using Subpathway Miner (13). A total of 3,925 associations were identified between 488 drugs and 403 subpathways in this study (12). These associations were downloaded and the intersecting subpathways between OS metastasis and the drugs were obtained for use in the present study. Among the 403 subpathways, 9 subpathways corresponding to 98 drugs were associated with OS metastasis. Detailed information and Anatomical Therapeutic Chemical classification for these drugs were obtained from the Drug Bank database (www.drugbank.ca).

Transwell invasion assay. U2OS cells (obtained from the American Type Culture Collection, Manassas, VA, USA) were starved through culturing in serum-free medium [RPMI-1640 (MGC-803); Gibco; Fisher Scientific, Inc., Waltham, MA, USA] for $12 \mathrm{~h}$ under standard conditions, as described by the American Type Culture Collection. The cells were subsequently seeded into 6 -well plates at a density of $2.5 \times 10^{5}$ cells/well. Following treatment with $100 \mu \mathrm{mol}$ lansoprazole for $24 \mathrm{~h}$ at $37^{\circ} \mathrm{C}$, cells were fixed and stained and their invasiveness was investigated using a Transwell chamber (Corning Incorporated, Corning,

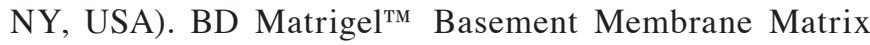
(50 mg/l; BD Biosciences, San Jose, CA, USA) was diluted with serum-free medium at a ratio of 1:8, and each Transwell chamber was coated with $60 \mu \mathrm{l}$ of this solution. Prior to use, the polycarbonate membrane (pore size, $8 \mu \mathrm{m}$ ) was hydrated with $50 \mu \mathrm{l}$ of serum-free medium containing $10 \mathrm{~g} / \mathrm{l}$ bovine serum albumin (Beyotime Institute of Biotechnology, Haimen, China) at $37^{\circ} \mathrm{C}$ for $30 \mathrm{~min}$. Treated cells $\left(8 \times 10^{5}\right.$ cells/well) were subsequently seeded into the upper chamber in $200 \mu \mathrm{l}$ of serum-free medium, and $600 \mu \mathrm{l}$ of medium containing $10 \%$ fetal bovine serum (Stemcell Technologies, Inc., Vancouver, $\mathrm{BC}$, Canada) was added to the lower chamber as an attractant. Following incubation for $24 \mathrm{~h}$ at $37^{\circ} \mathrm{C}$ in $5 \% \mathrm{CO}_{2}$, the cells that had invaded the lower surface of the filter were fixed with $75 \%$ ethanol and stained with $0.1 \%$ crystal violet. Cells were counted by eye in three random areas in each chamber (magnification, x200) using a light microscope.

Wound healing assay. The migration ability of U2OS cells was determined using a wound healing assay. U2OS cells were seeded into 6 -well plates $\left(2 \times 10^{5}\right)$. When the cells reached $70 \%$ confluence they were treated with lansoprazole $(200 \mu \mathrm{M})$. The control group was treated with DMSO.A sterile $10 \mu \mathrm{l}$ pipette tip was used to draw straight lines on the confluent monolayer. Photographs of the wounds were taken at 0 and $24 \mathrm{~h}$ post-treatment. The width of the wound at these time points was measured to measure cell migration.

Statistical analysis. All data were from $\geq 3$ independent experiments and are presented as the mean \pm standard deviation. The differences between groups were analyzed using the Student's t-test or one way analysis of variance with SPSS 19.0 software (IBM SPSS, Armonk, NY, USA) and Graph-Pad Prism 5.0 (GraphPad Software, Inc., La Jolla, CA, USA). P<0.05 was considered to indicate a statistically significance difference.

\section{Results}

DEG analysis between metastatic and non-metastatic OS samples. The microarray dataset GSE14827 from the GEO database was downloaded and used to identify DEGs between metastatic and non-metastatic OS samples. A total of 546 genes were identified as DEGs (data not shown). 
Table I. Enriched subpathway analysis.

\begin{tabular}{llr}
\hline Entire pathway & Subpathway Miner ID & P-value \\
\hline Steroid hormone biosynthesis $^{\mathrm{a}}$ & path:00140_17 & 0.00006 \\
Tyrosine metabolism $^{\mathrm{a}}$ & path:00350_12 & 0.01581 \\
Tyrosine metabolism $^{\mathrm{a}}$ & path:00350_4 & 0.01120 \\
Tyrosine metabolism $^{\mathrm{a}}$ & path:00350_5 & 0.02960 \\
Tyrosine metabolism $^{\mathrm{a}}$ & path:00350_6 & 0.04660 \\
Ether lipid metabolism $^{\mathrm{a}}$ & path:00565_4 & 0.01900 \\
Arachidonic acid metabolism $^{\mathrm{a}}$ & path:00590_6 & 0.02960 \\
Metabolism of xenobiotics by cytochrome P450 $^{\mathrm{a}}$ & path:00980_3 & 0.00221 \\
Drug metabolism-cytochrome P450 $^{\mathrm{a}}$ & path:00982_12 & 0.02960
\end{tabular}

${ }^{\mathrm{a}} \mathrm{P}<0.05$.

Investigating the underlying mechanism of OS metastasis based on subpathway enrichment analysis. To investigate the underlying mechanism of OS metastasis, dysregulated metabolic subpathways were identified through the use of Subpathway Miner (12). Following integration of the DEGs with metabolic subpathways $(\mathrm{k}=3), 9$ enriched metabolic subpathways corresponding to 6 entire metabolic pathways were identified (Table I). The 9 metabolic subpathways were considered significantly associated with the development of OS metastasis (all $\mathrm{P}<0.05$; Table I).

Drug repositioning for the treatment of metastatic OS. To determine if any existing drugs could be repositioned to treat metastatic OS, candidate small molecule drugs that were capable of targeting OS metastasis-associated metabolic subpathways were identified. The global associations between drugs and metabolic subpathways were downloaded from a study performed by Li et al (12), in which 403 metabolic subpathways affected by 488 known drugs were identified through the use of Subpathway Miner $(\mathrm{k}=3)$.

A total of 9 overlapping subpathways associated with OS metastasis and affected by small molecule drugs were identified. In addition, 98 small molecule drugs were identified in these subpathways (Table II). Detailed information and Anatomical Therapeutic Chemical classification for these drugs were obtained from the Drug Bank database (www.drugbank.ca).

A bipartite network of the drugs identified and the overlapping metabolic subpathways was built (Fig. 1). In this network, certain drugs could affect several metabolic subpathways. For example, lansoprazole and omeprazole perturbed 8 and 7 subpathways, respectively, while others affected fewer subpathways.

Lansoprazole inhibits the invasiveness of U2OS cells. In order to investigate the effect of the drugs identified on the invasiveness of OS cells, a Transwell assay was performed on U2OS cells following treatment with lansoprazole, due to it affecting the most subpathways. As illustrated in Fig. 2, lansoprazole significantly inhibited the invasion of $20 \mathrm{OS}$ cells compared with the control group $(\mathrm{P}=0.02)$ and did so in a dose-dependent manner. Furthermore, cell migration was assessed using a wound healing assay. The results of this assay demonstrated a marked reduction in cell migration following lansoprazole treatment (Fig. 3).

\section{Discussion}

Microarray analysis using high-throughput screening technology is an important tool for studying gene expression patterns. In addition, this tool can be used to identify potential therapeutic targets for improving therapeutic interventions. In the present study, the gene expression profiles of biopsy specimens from patients who did or did not develop metastatic OS were used to investigate the mechanisms underlying the development of metastatic OS. A total of 546 DEGs were identified between the two groups. Results from Subpathway Miner analysis demonstrated that 9 metabolic subpathways corresponding to 6 entire pathways were associated with OS metastasis. A total of 98 candidate small molecule drugs involved in the regulation of OS metastasis were subsequently identified through integrating OS metastasis-associated and drug-affected subpathways.

Subpathway analysis focuses on a specific area of a pathway, rather than the entire pathway. Thus, it can identify more subtle subpathways that may be neglected through the analysis of entire pathways, and may be more suitable and flexible compared with entire pathway analysis for the identification of disease mechanisms and drug responses $(12,13)$. In the present study, 9 subpathways corresponding to 6 entire KEGG metabolic pathways were identified. A number of these subpathways have been revealed to serve important roles in OS metastasis, such as steroid hormone biosynthesis (path:00140_17; $\mathrm{P}=6.10 \times 10^{-5}$ ). A report by Fang et al (14) indicated that various concentrations of estrogen lead to changes in several physiological processes in OS, such as cell proliferation, migration, invasion and epithelial-mesenchymal transition. In addition, the enzymes (Cyclooxygenase 1 and 2) that are essential for arachidonic acid metabolism (path:00590_6; $\mathrm{P}=0.029560322$ ) have been identified to be involved in the apoptosis of OS cells $(15,16)$. Furthermore, 4/9 subpathways originated from the tyrosine metabolism pathway (path:00350), which has been demonstrated to influence the growth of OS cells (17).

In the present study, a group of known drugs with potential therapeutic efficacy for OS metastasis were identified. A total of 98 small molecule drugs that have common subpathways with OS metastasis were identified, including a number of anti-cancer drugs. For example, semustine, a chloroethyl nitrosourea, is known to be therapeutically effective against murine models of 
Table II. Candidate small molecule drugs for the treatment of metastatic osteosarcoma.

\begin{tabular}{|c|c|c|c|}
\hline Drug name & $\begin{array}{l}\text { No. of overlapping } \\
\text { subpathways involved in }\end{array}$ & $\begin{array}{c}\text { ATC } \\
\text { classification }\end{array}$ & Drug information \\
\hline Lansoprazole & 8 & $\mathrm{~A} 02 \mathrm{BC} 03$ & Proton pump inhibitor \\
\hline Omeprazole & 7 & $\mathrm{~A} 02 \mathrm{BC} 01$ & Proton pump inhibitor \\
\hline Phentolamine & 6 & C04AB01 & Imidazoline derivative \\
\hline Amodiaquine & 2 & P01BA06 & Aminoquinoquinoline \\
\hline Apomorphine & 2 & G04BE07 & Drug used in erectile dysfunction \\
\hline Butamben & 2 & & \\
\hline Carbamazepine & 2 & N03AF01 & Carboxamide derivative \\
\hline Cefalonium & 2 & & \\
\hline Clioquinol & 2 & & \\
\hline Colforsin & 2 & & \\
\hline Etanidazole & 2 & & \\
\hline Isotretinoin & 2 & D10AD04 & Retinoid for topical use in acne \\
\hline Leflunomide & 2 & L04AA13 & Selective immunosuppressant \\
\hline Lisuride & 2 & G02CB02 & Prolactine inhibitor \\
\hline Mefloquine & 2 & $\mathrm{P} 01 \mathrm{BC} 02$ & Methanolquinoline \\
\hline Megestrol & 2 & & \\
\hline Methoxsalen & 2 & D05AD02 & Psoralen for topical use \\
\hline Nabumetone & 2 & M01AX01 & $\begin{array}{l}\text { Other anti-inflammatory and anti-rheumatic agents, } \\
\text { non-steroidal }\end{array}$ \\
\hline Naringenin & 2 & & \\
\hline Phenazopyridine & 2 & G04BX06 & Other urological agent \\
\hline Phenelzine & 2 & N06AF03 & Monoamine oxidase inhibitor, non-selective \\
\hline Pipenzolate bromide & 2 & & \\
\hline Progesterone & 2 & G03DA04 & Pregnen derivative \\
\hline Raubasine & 2 & & \\
\hline Riboflavin & 2 & & \\
\hline Risperidone & 2 & N05AX08 & Other antipsychotic \\
\hline Sulindac & 2 & M01AB02 & Acetic acid derivative and related substances \\
\hline Tetracycline & 2 & A01AB 13 & Anti-infective and antiseptic for local oral treatment \\
\hline Tiapride & 2 & & \\
\hline Trioxysalen & 2 & & \\
\hline Yohimbine & 2 & G04BE04 & Drug used in erectile dysfunction \\
\hline Astemizole & 1 & R06AX11 & Other antihistamine for systemic use \\
\hline Atracurium besilate & 1 & & \\
\hline Azapropazone & 1 & M01AX04 & $\begin{array}{l}\text { Other anti-inflammatory and anti-rheumatic agent, } \\
\text { non-steroidal }\end{array}$ \\
\hline Aztreonam & 1 & J01DF01 & Monobactam \\
\hline Carmustine & 1 & L01AD01 & Nitrosourea \\
\hline Chenodeoxycholic acid & 1 & A05AA01 & Bile acid preparation \\
\hline Dinoprostone & 1 & G02AD02 & Prostaglandin \\
\hline Diphenhydramine & 1 & D04AA32 & Antihistamine for topical use \\
\hline Disulfiram & 1 & N07BB01 & Sulfur containing product \\
\hline Dosulepin & 1 & & \\
\hline Dyclonine & 1 & N01BX02 & Other local anesthetic \\
\hline Econazole & 1 & D01AC03 & Imidazole and triazole derivative \\
\hline Etacrynic acid & 1 & $\mathrm{C} 03 \mathrm{CC} 01$ & Aryloxyacetic acid derivative \\
\hline Ethosuximide & 1 & N03AD01 & Succinimide derivative \\
\hline Etilefrine & 1 & & \\
\hline Fenspiride & 1 & R03BX01 & Other drug for obstructive airway diseases, inhalant \\
\hline Gliquidone & 1 & A10BB08 & Sulfonylurea \\
\hline Heptaminol & 1 & & \\
\hline
\end{tabular}


Table II. Continued.

\begin{tabular}{|c|c|c|c|}
\hline Drug name & $\begin{array}{c}\text { No. of overlapping } \\
\text { subpathways involved in }\end{array}$ & $\begin{array}{c}\text { ATC } \\
\text { classification }\end{array}$ & Drug information \\
\hline Homochlorcyclizine & 1 & & \\
\hline Hydralazine & 1 & C02DB02 & Hydrazinophthalazine derivative \\
\hline Imipenem & 1 & & \\
\hline Ketorolac & 1 & M01AB 15 & Acetic acid derivative and related substances \\
\hline Lymecycline & 1 & J01AA04 & Tetracycline \\
\hline Meteneprost & 1 & & \\
\hline Meticrane & 1 & & \\
\hline Metrifonate & 1 & & \\
\hline Monobenzone & 1 & D11AX13 & Other dermatological \\
\hline Moracizine & 1 & C01BG01 & Other antiarrhythmic, class I and III \\
\hline Myricetin & 1 & & \\
\hline Nadide & 1 & & \\
\hline Netilmicin & 1 & J01GB07 & Other aminoglycoside \\
\hline Nifedipine & 1 & C08CA05 & Dihydropyridine derivative \\
\hline Nifenazone & 1 & & \\
\hline Nifuroxazide & 1 & & \\
\hline Norfloxacin & 1 & J01MA06 & Fluoroquinolone \\
\hline Nortriptyline & 1 & N06AA10 & Non-selective monoamine reuptake inhibitor \\
\hline Oxybenzone & 1 & & \\
\hline Oxyphenbutazone & 1 & & \\
\hline Ozagrel & 1 & & \\
\hline Pentoxifylline & 1 & C04AD03 & Purine derivative \\
\hline Phenoxybenzamine & 1 & $\mathrm{C} 04 \mathrm{AX} 02$ & Other peripheral vasodilator \\
\hline Pioglitazone & 1 & A10BG03 & Thiazolidinedione \\
\hline Pivmecillinam & 1 & J01CA08 & Penicillin with extended spectrum \\
\hline Pizotifen & 1 & & \\
\hline Praziquantel & 1 & P02BA01 & Quinoline derivative and related substances \\
\hline Prochlorperazine & 1 & N05AB04 & Phenothiazine with piperazine structure \\
\hline Profenamine & 1 & & \\
\hline Proxyphylline & 1 & & \\
\hline Quercetin & 1 & & \\
\hline Ribavirin & 1 & J05АВ04 & Nucleosides and nucleotides \\
\hline Rilmenidine & 1 & & \\
\hline Rimexolone & 1 & H02AB 12 & Glucocorticoid \\
\hline Ritodrine & 1 & G02CA01 & Sympathomimetic, labor repressant \\
\hline Salbutamol & 1 & $\mathrm{R} 03 \mathrm{AC} 02$ & Selective $\beta 2$-adrenoreceptor agonist \\
\hline Semustine & 1 & & \\
\hline Sertaconazole & 1 & D01AC14 & Imidazole and triazole derivative \\
\hline Simvastatin & 1 & C10AA01 & HMG-CoA reductase inhibitor \\
\hline Tanespimycin & 1 & & \\
\hline Terbutaline & 1 & $\mathrm{R} 03 \mathrm{AC} 03$ & Selective $\beta 2$-adrenoreceptor agonist \\
\hline Thalidomide & 1 & L04AX02 & Other immunosuppressant \\
\hline Tiabendazole & 1 & D01AC06 & Imidazole and triazole derivative \\
\hline Tiletamine & 1 & & \\
\hline Tracazolate & 1 & & \\
\hline Tranylcypromine & 1 & N06AF04 & Monoamine oxidase inhibitor, non-selective \\
\hline Tretinoin & 1 & D10AD01 & Retinoid for topical use in acne \\
\hline Triamterene & 1 & C03DB02 & Other potassium-sparing agent \\
\hline Troglitazone & 1 & & \\
\hline
\end{tabular}

ATC, Anatomical Therapeutic Chemical; HMG-CoA, 3-hydroxy-3-methylglutaryl-coenzyme A. 


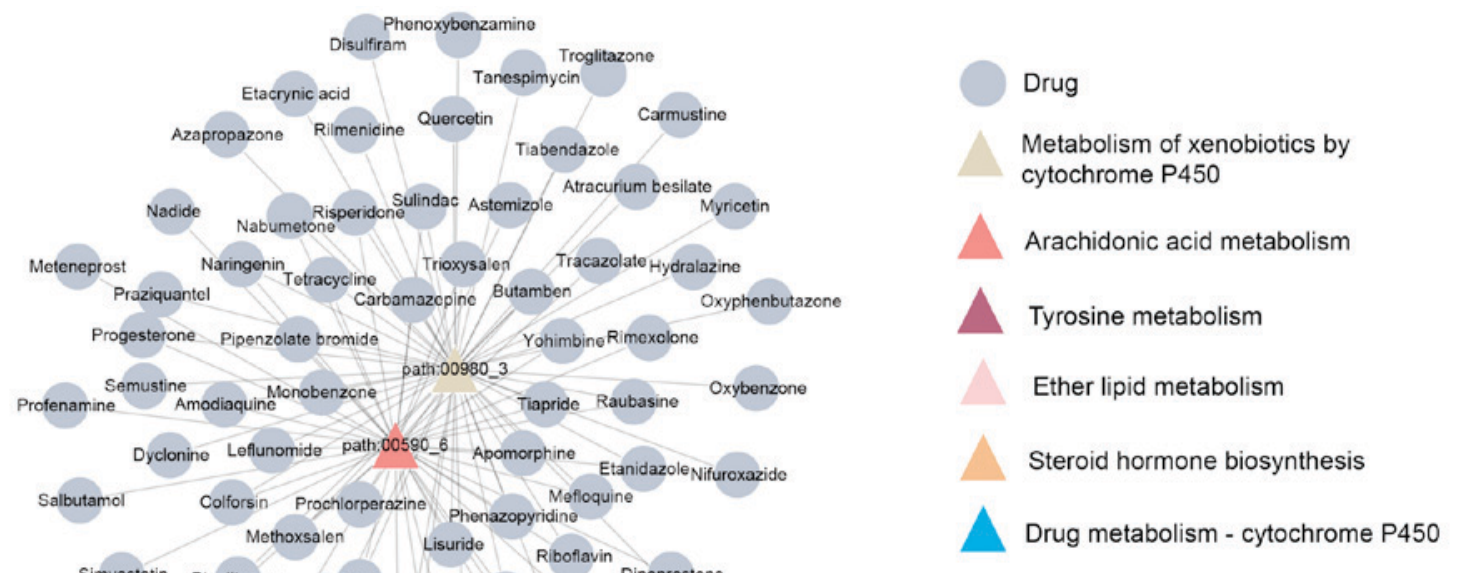

Figure 1. Small molecule drugs and their corresponding subpathways. Triangular nodes represent subpathways and circular nodes represent drugs. Subpathways of the same color are included in the same entire pathway.
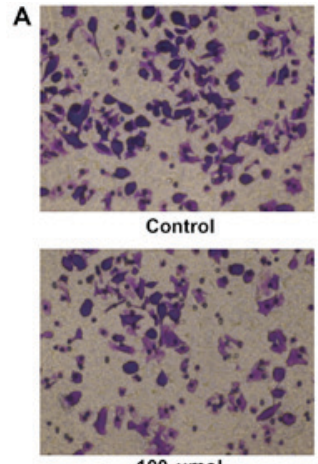

$100 \mu \mathrm{mol}$
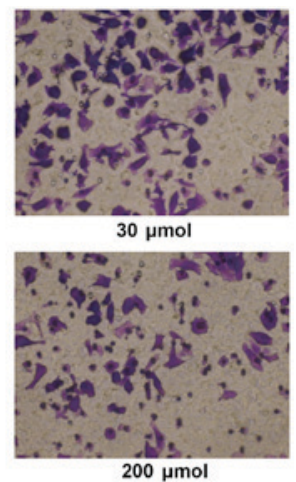

B

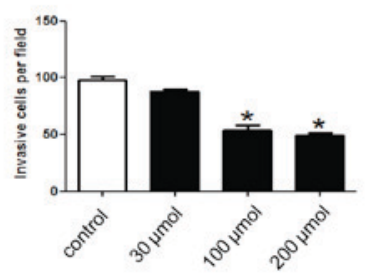

Figure 2. U2OS cell invasion following lansoprazole treatment was assessed using the Transwell assay. (A) Representative images of invasive cells (magnification, x200). (B) Relative levels of invasive cells compared with the control. ${ }^{*} \mathrm{P}<0.05$ vs. the control group.

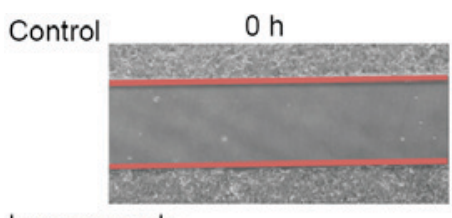

\section{Lansoprazole}
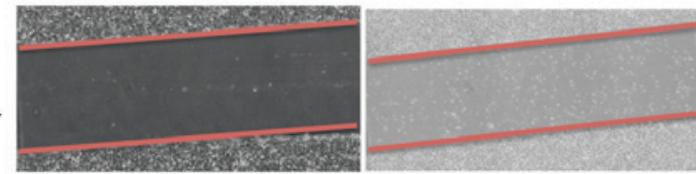

Figure 3. Effect of lansoprazole $(100 \mu \mathrm{M})$ on the migration of cultured U2OS cells, assessed through a wound healing assay.

several tumor types (18-20). In addition, a number of the drugs identified have been proven to possess potential anti-OS effects. For example, progesterone may inhibit the proliferation of OS cells and increase the expression of c-Fos and c-Jun (21). Notably, the proton pump inhibitor lansoprazole, which could influence 8/9 OS metastasis-associated subpathways in the current study, has not, to the best of our knowledge, been demonstrated to modulate the metastasis of OS. However, lansoprazole has been 
revealed to contribute to proliferation and invasion of breast cancer cells during tumorigenesis and metastasis (22).

To further investigate the effects of lansoprazole on OS metastasis, OS U2OS cells were treated with different concentrations of lansoprazole. Lansoprazole was demonstrated to inhibit invasion of U2OS cells in a dose-dependent manner. In addition, a wound healing assay demonstrated that lansoprazole markedly decreased U2OS cell migration. In conclusion, the present study presents a bioinformatics approach based on subpathway analysis to identify potential agents that regulate OS metastasis, such as lansoprazole. However, further experiments are warranted to investigate the safety of lansoprazole and explore the underlying molecular mechanisms of its effects on OS metastasis.

\section{References}

1. He H, Ni J and Huang J: Molecular mechanisms of chemoresistance in osteosarcoma (Review). Oncol Lett 7: 1352-1362, 2014.

2. Chou AJ and Gorlick R: Chemotherapy resistance in osteosarcoma: Current challenges and future directions. Expert Rev Anticancer Ther 6: 1075-1085, 2006.

3. Longhi A, Errani C, De Paolis M, Mercuri M and Bacci G: Primary bone osteosarcoma in the pediatric age: State of the art. Cancer Treat Rev 32: 423-436, 2006.

4. He JP, Hao Y, Wang XL, Yang XJ, Shao JF, Guo FJ and Feng JX: Review of the molecular pathogenesis of osteosarcoma. Asian Pac J Cancer Prev 15: 5967-5976, 2014.

5. Broadhead ML, Clark JC, Myers DE, Dass CR and Choong PF: The molecular pathogenesis of osteosarcoma: A review. Sarcoma 2011: 959248, 2011.

6. Bielack SS, Kempf-Bielack B, Delling G, Exner GU, Flege S, Helmke K, Kotz R, Salzer-Kuntschik M, Werner M, Winkelmann W, et al: Prognostic factors in high-grade osteosarcoma of the extremities or trunk: An analysis of 1,702 patients treated on neoadjuvant cooperative osteosarcoma study group protocols. J Clin Oncol 20: 776-790, 2002.

7. Kobayashi E, Masuda M, Nakayama R, Ichikawa H, Satow R, Shitashige M, Honda K, Yamaguchi U, Shoji A, Tochigi N, et al: Reduced argininosuccinate synthetase is a predictive biomarker for the development of pulmonary metastasis in patients with osteosarcoma. Mol Cancer Ther 9: 535-544, 2010.

8. Hughes DP: Strategies for the targeted delivery of therapeutics for osteosarcoma. Expert Opin Drug Deliv 6: 1311-1321, 2009.

9. Bielack SS, Carrle D, Hardes J, Schuck A and Paulussen M: Bone tumors in adolescents and young adults. Curr Treat Options Oncol 9: 67-80, 2008.
10. Osaki S, Tazawa H, Hasei J, Yamakawa Y, Omori T, Sugiu K, Komatsubara T, Fujiwara T, Sasaki T, Kunisada T, et al: Ablation of MCL1 expression by virally induced microRNA-29 reverses chemoresistance in human osteosarcomas. Sci Rep 6: 28953, 2016.

11. Lamb J, Crawford ED, Peck D, Modell JW, Blat IC, Wrobel MJ, Lerner J, Brunet JP, Subramanian A, Ross KN, et al: The connectivity map: Using gene-expression signatures to connect small molecules, genes, and disease. Science 313: 1929-1935, 2006.

12. Li C, Li X, Miao Y, Wang Q, Jiang W, Xu C, Li J, Han J, Zhang F, Gong B and Xu L: SubpathwayMiner: A software package for flexible identification of pathways. Nucleic Acids Res 37: e131, 2009.

13. Li C, Shang D, Wang Y, Li J, Han J, Wang S, Yao Q, Wang Y, Zhang Y, Zhang C, et al: Characterizing the network of drugs and their affected metabolic subpathways. PLoS One 7: e47326, 2012.

14. Fang D, Yang H, Lin J, Teng Y, Jiang Y, Chen J and Li Y: $17 \beta$-estradiol regulates cell proliferation, colony formation, migration, invasion and promotes apoptosis by upregulating miR-9 and thus degrades MALAT-1 in osteosarcoma cell MG-63 in an estrogen receptor-independent manner. Biochem Biophys Res Commun 457: 500-506, 2015.

15. Moalic S, Liagre B, Corbière C, Bianchi A, Dauça M, Bordji K and Beneytout JL: A plant steroid, diosgenin, induces apoptosis, cell cycle arrest and COX activity in osteosarcoma cells. FEBS Lett 506: 225-230, 2001.

16. Moalic S,Liagre B,Le Bail JC and Beneytout JL: Dose-dependent modulation of apoptosis and cyclooxygenase-2 expression in human 1547 osteosarcoma cells by NS-398, a selective cyclooxygenase-2 inhibitor. Int J Oncol 18: 533-540, 2001.

17. Tinti L, Taylor AM, Santucci A, Wlodarski B, Wilson PJ, Jarvis JC, Fraser WD, Davidson JS, Ranganath LR and Gallagher JA: Development of an in vitro model to investigate joint ochronosis in alkaptonuria. Rheumatology 50: 271-277, 2011.

18. Wang X, Chen JX, Liu JP, You C, Liu YH and Mao Q: Gain of function of mutant TP53 in glioblastoma: Prognosis and response to temozolomide. Ann Surg Oncol 21: 1337-1344, 2014.

19. Saha P, Debnath C and Bérubé G: Steroid-linked nitrogen mustards as potential anticancer therapeutics: A review. J Steroid Biochem Mol Biol 137: 271-300, 2013.

20. Bartzatt R: Anticancer agents for treatment of tumors in the central nervous system by correspondent substituent substitution and elucidation by pattern recognition methods. Med Chem 8: 138-144, 2012.

21. Liang M, Liao EY, Xu X, Luo XH and Xiao XH: Effects of progesterone and 18-methyl levonorgestrel on osteoblastic cells. Endocr Res 29: 483-501, 2003.

22. Zhang S, Wang Y and Li SJ: Lansoprazole induces apoptosis of breast cancer cells through inhibition of intracellular proton extrusion. Biochem Biophys Res Commun 448: 424-429, 2014. 